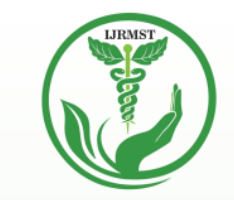

INTERNATIONAL JOURNAL OF RESEARCH IN MEDICAL SCIENCES \& TECHNOLOGY

e-ISSN:2455-5134; p-ISSN: 2455-9059

\title{
ATTEMPTING AN INDEPTH ANALYSIS OF RETINAL IMAGE SEGMENTATION
}

\section{Prachi Juneja}

Sri Guru Gobind Singh College Of Commerce, University Of Delhi

Paper Received: 27 $7^{\text {th }}$ April, 2021; Paper Accepted: 24 ${ }^{\text {th }}$ May, 202 1;

Paper Published: $29^{\text {th }}$ June, 2021

DOI: http://doi.org/10.37648/ijrmst.vl li01.009

How to cite the article:

Prachi Juneja, Attempting an in-depth Analysis of Retinal Image

Segmentation, IJRMST, January-June 2021, Vol 11, 100-119, DOI: http://doi.org/10.37648/ijrmst.v11i01.009

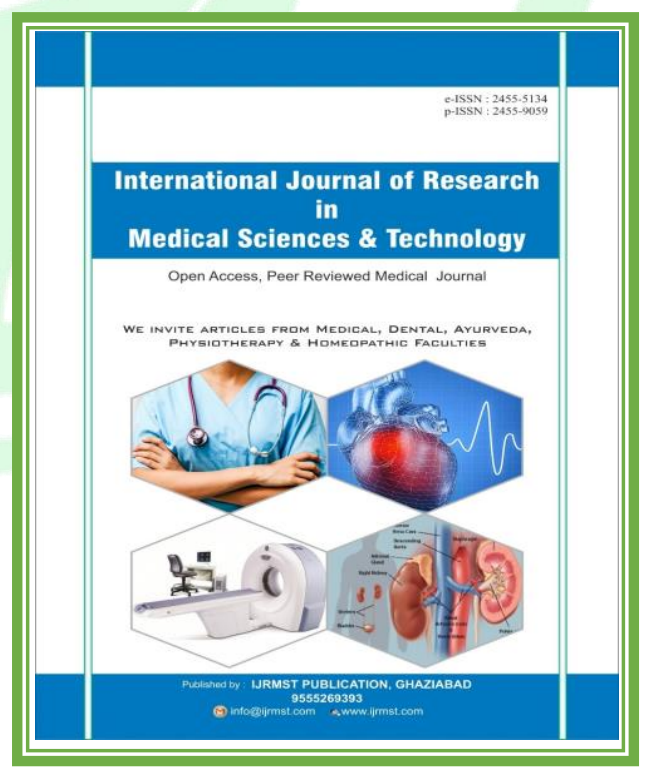




\begin{abstract}
These days eye weaknesses are a typical issue in all age group individuals begins from a newborn child to mature age. The discovery and extraction of these infections is a troublesome and tedious assignment. Computerized retinal pictures are considered; the first important strategy is to separate vessel in fundus pictures. Thus, three methods are utilized regulated techniques; here, the training set applies to remove vessel data by the pre-trained algorithm. This strategy is physically dealt with using gold std; vessel extraction is done before pathology calculations are involved in unaided recognition and extraction programs. The preparation set and ground truth marking will not be straightforwardly appropriate to the analysis. Retinal vessels extraction is improving as a result of noninvasive imaging of the retinal pictures likewise the information got from the design of the vasculature, and this data is essential for the identification and analysis of a fundus picture retinal sicknesses and pathologies, which incorporates glaucoma, hypertension, Diabetics Retina chart, and Age-based Macula De-age. Quick division calculations can recognize these.
\end{abstract}

Watchwords: Age-based Macula De-age; Diabetics Retina diagram; Diabetics macula oedema; Glaucoma; Hyper-strain

\section{INTRODUCTION}

Eye related vessels identification and extraction are the major obsessive issue in the field of ophthalmology. The aetiology of vessel sicknesses is as yet an examination region where much innovative work is in progress. The ID, extraction, and limitation of the retinal fundus and retinal pictures in different organizations intend to isolate the distinctive retina arch and vasculature tissues, including broadened and compact designs inside the retina fundus retinal foundation pictures and other optical constructions like optic-circle, exudates, macular, fovea and injury. Innovative work of over twenty years, the strategies with inventive and quick methodologies applies with automated techniques related to restricting and division in retinal eye vessels like design are significant, and it's getting essential and essential to constant clinical applications. The body part picture eye retinal vessel portion techniques have some normal handling stages beginning with preprocessing, mid-preparing, and post-handling steps. The survey sorts the essentials of the method utilized, calculation stream and specialized strategies applied in each location. The administered calculation techniques expand on pre-arrange information; the effectiveness of the managed system is better than un-regulated strategies, which produce precise outcomes to great fundus 


\section{VOLUME: 1 1, JANUARY-JUNE 2021}

retina eye images. These driven and solo strategies are the most commonly utilized picture division techniques. Fundamentally describes the accompanying five algorithms for investigation. The first is the limit division strategy. This technique is most usually used in area based division calculations [2]. Its utilization consequently removes the standardized edge esteem dependent on the specific model and applies these pixels esteems as indicated by the greyscale level to accomplish required grouping. The general territorial development procedure can combine the pixel values with similar properties to get the locale. The Edge discovery division calculation utilizes different districts of the image component dim level or shading level irregularity in the identification space of the edge to accomplish fundus picture division [3]. In clustering division, setting a name to each picture component in the retinal picture comprises three locales. The first is a picture that contains objects. Besides, give the item line. Thirdly object territory in the image is set apart with a partial picture component [5]

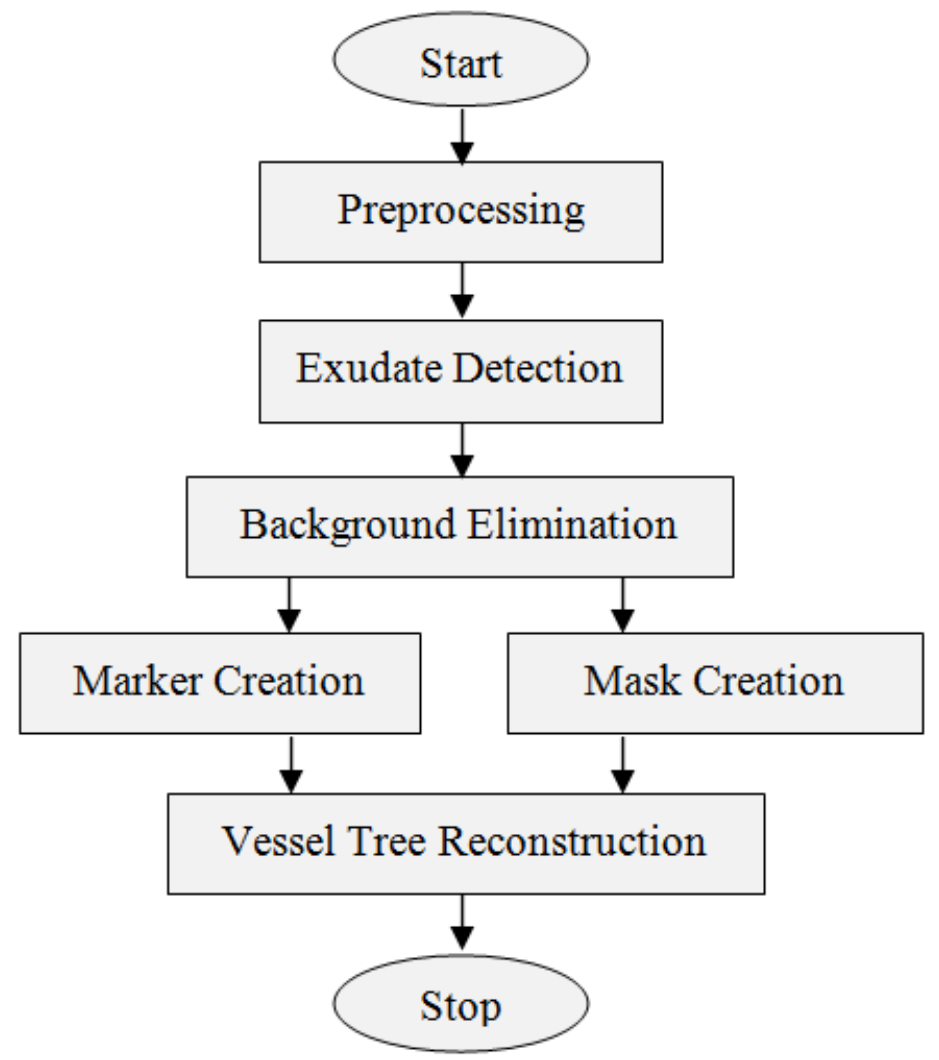

Figure 1: General vessel extraction algorithm 


\section{VOLUME: 1 1, JANUARY-JUNE 2021}

The potential and the capacity of retinal and fundus image portion or disintegrate techniques are to passage or eliminate the eye vascular and supported by so many cadent. The generally applied boundaries are: mean Truly-Positive-Rate (TPR), average Falsely Positive Rate (FPR), moderate-Sensitivity, mean-Specificity, mean-Accuracy, and precision. Delicate and explicit boundaries are the most commonly utilized evaluations in ophthalmology. Assuming qualities are higher for these boundaries, the treatment is better. The risky boundary ruminates the capacity of this technique to see eye vessels picture components; the particular boundaries examine the fitness of the strategy to separate no-vessel components. Explicitness and explicit boundaries reflect featuring of leap forward, which is identified with the exact worth in some allopathic eye retina fundus imaging. For the most part, the vessel extraction calculation applies the accompanying three boundaries for the specific extraction of vessels. Affectability $=T P T P+F N$ Specificity $=T N T N+F P \quad$ Accuracy $=T P+T N T P+F N+T N+F P$ Where TP: True Positives: vessel pixels which are effectively arranged. FP, False Positives: non-vessel pixels which are wrongly delegated vessel pixels. TN: True Negatives: non-vessel pixels which are effectively grouped. FN: False Negatives: vessel pixels that are incorrectly named non-vessel pixels. Affectability: Ratio of accurately arranged vessel pixels and the all outnumber of vessel pixels. Particularity: Ratio of effectively grouped non-vessel pixels and the complete number of non-vessel pixels. Precision: Extent to which calculation works effectively.

\section{LITERATURE SURVEY}

The retinal images various process explained

\section{A. Preprocessing on Retina Image}

The main issue experienced by clinical imaging frameworks is the twisting of visual signs acquired because of defective procurement and transmission mistakes. Visible bending may emerge because of different elements like a season of openness, lighting, and development of the eye and affectability of the imaging gadgets. These influence pictures as far as differentiation, twisting and ancient rarities presented, obscure and contrast affectability. These visual changes have an adverse consequence and make the picture complex for understanding. This requires picture upgrade strategies that improve quality boundaries. Models incorporate histogram balance, picture honing, contrast change, edge upgrade and denoising. 


\section{VOLUME: 1 1, JANUARY-JUNE 2021}

Picture preprocessing procedures include picture contrast improvement, picture commotion evacuation, edge, edge identification and picture division.

Picture pre-preparing is the essential technique of computerized fundus picture. Clamour in the clinical images has two impediments. They debase the picture quality and dark the critical data needed for exact analysis. Both have a natural effect during the investigation and must be dealt with productively. In this manner, all clinical pictures need a calculation to upgrade the image and assist the clinical professional with diagnosing and productively.

Marco et al. (2005) [6] has utilized the pay based procedure for killing the glow and difference varieties in the retinal pictures. Standardization of these varieties is performed by assessing the nonconsistency behind the scenes part of the picture. In any case, not many zones with "non-foundation" dim regions bigger than half are not influenced by this standardization.

Aliaa et al. (2006) [7] has introduced a similar report between different differentiation upgrade strategies for retinal pictures. These strategies are carried out on openly accessible data sets, and the outcomes are classified. These outcomes dissect the benefits and negative marks of the different differentiation upgrade strategies.

Peng et al. (2007) [8] has utilized change based strategies for edge improvement in low differentiation pictures. The yield pictures depend on the non-straight capacity, which joins the impacts of clamour. A similar examination is additionally performed with different procedures. The meaning of the red channel for precise shading retinal picture handling is clarified by Nancy et al. (2007).

Division of retinal veins is performed with red and green channels of the retinal picture. The outcomes are contrasted and the dividend yield of the green track of the retinal image. Experimental results have shown promising effects for the utilization of the red channel for retinal picture division. A close examination of the prepreparing methods in shading retinal pictures is performed by Salvatelli et al. (2007) [9].

George et al. (2008) [10] has carried out a subordinate based strategy for foundation closer view separation. The convolution of 2D Gaussian portions with the second subordinates of the info picture features the veins. However, the disadvantage of 


\section{VOLUME: 1 1, JANUARY-JUNE 2021}

this framework is the low precision results determined in the report.

Gopal et al. (2008) [11] has proposed an area information based vein improvement strategy in shading retinal pictures. A remedy factor is gotten from the assessed corruption and used to limit the difference and iridescence variety in retinal images. A model-based vessel improvement method is proposed by Yuan et al. (2008) [12]. The foundation concealment measure, smoother 'vesselness' measure and the reactions at intersections are better than the traditional strategies. The directional field-based retinal vessel upgrade procedure is accounted for by Jian et al. (2008) [13]. A solitary advance brilliance standardization and neighbourhood improvement are utilized in this work.

Multi-scale line activity-based vein improvement is performed by Farnell et al. (2008) [14]. This multi-scale line activity calculation depends on the district developing strategy, and the outcomes are contrasted and the traditional middle sifting method. The proposed approach is appropriate for all retinal infection grouping applications, and the effects likewise uncovered that the proposed way is a lot quicker than the traditional procedures.
During the past many years, numerous strategies have been proposed to deal with the clinical picture division issues utilizing solo bunching techniques. Nonetheless, the systems have a few restrictions to defeat the picture's problems, like force inhomogeneity, fractional volume impact, substantial commotions and different relics during the division cycle. Among any remaining numerical based division strategies, Fuzzy C-Means has gotten a lot of consideration since it acquires the first data from the actual picture. It doesn't need any earlier data to handle the division framework. Fluffy C-Means is the strategy for the soft bunching method. Fluffy grouping is quite possibly the main procedures in bunch investigation. Throughout the long term, there have been numerous strategies and techniques created to perform group examination. Most conventional bunch examination strategies are fresh parcelling, in which each given article is rigorously characterized into a specific gathering. Notwithstanding, practically speaking, the class ascribes of most papers are not severe and uncertain; subsequently, it isn't reasonable for hard apportioning. Favourably, the fluffy set hypothesis was proposed by Lotfi. A. Zadeh [15]. It augments the traditional set hypothesis, and it gives a valuable asset to delicate parcelling. Utilizing fluffy set 


\section{VOLUME: 1 1, JANUARY-JUNE 2021}

hypothesis [5] for bunching is first presented by Ruspini [15]. Since fluffy bunching acquires the level of vulnerability of tests assets to each class and communicates the moderate property of their enrollments, it can all the more equitably mirror this present reality issues. As of late, the Fuzzy bunching strategy [16] is generally applied in numerous applications like the clinical conclusion, design acknowledgement, information examination and picture division. Since it doesn't need any earlier data about the objects of information and any human obstruction of images, it is a significant apparatus in dissecting pictures' conduct and underlying intricacy in clinical picture division [17]. The fluffy grouping measure is done viably by utilizing the FCM calculation [18].

B. Retinal Images template matching and its registration

A few works on layout coordinating of retinal pictures depend on more broad picture enrollment techniques, extensively concentrated as of late. Notwithstanding, general retina enrollment techniques centre around coordinating with picture sets with an enormous FOV with neighbourhood disfigurements or diverse picture modalities. The current retinal format corresponding with calculations are from the picture. The layout consistently contains a specific element, like the optic circle, exudate and antiques [19]-[20].

Retinal picture enrollment itself is testing: the nonvascular surface of the retina is homogeneous in solid retinas while displaying an assortment of pathologies in undesirable retinas [21]. Retina pictures caught by connector based optics give fewer data and have low picture quality, further expanding the trouble of layout coordinating. It is informative to present current retina picture enlistment techniques, which can use for format corresponding and their achievability intending to our expressed issue. Retina picture enlistment approaches can be grouped into territory based and include based techniques. Highlight based strategies upgrade the correspondence between removed notable items in retina pictures [21]. Regularly, bifurcations, fovea, and the optic circle are essential highlights utilized for retinal picture enrollment. A little FOV layout has little likelihood of containing explicit tourist spots on the retina. Subsequently, the fovea and optic circle are not appropriate. Vascular bifurcations are more regular, while correspondingly, the modest number of bifurcations in the format can't shape the premise of a hearty enrollment. Also, 


\section{VOLUME: 1 1, JANUARY-JUNE 2021}

the extraction of the vascular organization in low-quality pictures is troublesome. It can cause questionable vascular bearings while marking the bifurcations. General element point-based methodologies are additionally executed in retina enlistment, for example, SIFT-based [22] and SURF based techniques [23]. These methodologies can enrol the pictures in complex situations and are computationally proficient. They accept the element point sets can be dependably distinguished and coordinated to appraise the change. Albeit achievable by and large, the interaction can fizzle on bad quality retina pictures without definite highlights. Territory based methodologies match the force contrasts of a picture pair under a similitude measure, like SSD (amount of squared differences) [24], CC (CrossCorrelation) [25] and MI (common data) [26], at that point improve the comparability measure via looking in the change space. Staying away from pixellevel element recognition, such methodologies are more hearty to lowquality pictures than include based methods. Be that as it may, retina pictures with meagre highlights and comparable foundations will probably lead the advancement into neighbourhood extrema.
Since ophthalmology is mainly subject to visual data, it is an ideal claim to fame for telemedicine [27]. Advanced catch of pictures and the potential for transmission of these pictures through electronic exchange across enormous distances with resulting picture examination offers adequate utilization of clinical assets in massive, rustic networks that may somehow experience issues getting wellqualified assessment [28]. The most widely recognized framework used in 'teleophthalmology is "store-and-forward", where pictures are caught and later sent electronically to be examined later. These differentiations with live videoconferencing, which is right now restricted by electronic transmission rates. "Teleophthalmology" could be used between essential medical services experts, optometrists and ophthalmic trained professionals, or between various ophthalmic units. Telemedicine has even been utilized to help clinical jail officials diagnose ophthalmic objections, diminishing expenses and possible inconveniences of detainees going to expert clinical focuses [29]. In a communitarian worldwide venture, telemedicine is financially savvy in reducing the weight of eye sickness. More extravagant nations may help limit working in the medical services 
frameworks of less fortunate countries.

Nations with enormous spaces of meagerly populated networks, for example, Canada, Australia and India, may significantly profit by medical care conveyance to these spaces.

Telemedicine has a possible part in diabetic screening. Kawasaki et al. (2003) [30] report that 1076 of 1170 eyes' fundal pictures were effectively assessed by a specialist ophthalmologist when moved images through electronic mail. Lin et al. (2002) [31] report single non-mydriatic monochromatic wide-field computerized photography of the circle and macula to be more delicate for diabetic retinopathy screening mydriatic ophthalmoscopy when communicated electronically to an understanding site. When mediated by standard seven-field shading photos, the higher affectability of advanced photography mirrored the diminished affectability of ophthalmoscopy in distinguishing early retinopathy. TOSCA (Tele-Ophthalmological Services CitizenCentered Application) was created in Europe as a task to decrease the rate of visual impairment brought about by diabetic retinopathy (Luzio et al., 2004) [32]. Telemedicine has been investigated in evaluating for ROP. Yen et al. (2002) [33] discovered RetCam (Massie Research Laboratories, Inc., Dublin, CA) pictures caught by a neonatal attendant contrasted well and assessments performed by an accomplished ophthalmologist with great affectability, however, just reasonable particularity.

Furthermore, teleophthalmology has been used in macular sicknesses.

Eikelboom et al. (2000) [34] report on JPEG and wavelet advanced picture pressure on the nature of pictures for telemedicine. JPEG picture pressure breaks the image into squares of $8 \times 8$ pixels and converts these squares into spatial recurrence parts. Inspecting this recurrence space data by intently safeguarding the low-recurrence details and approximating the high-recurrence segments is performed. The measure of data disposed of decides the measurement of pressure. Wavelet utilizes band channels and low pass channels to the pixel lines and features of a picture. This produces data on the low-recurrence segments of the image and the even, vertical and inclining subtlety in the picture (and is all the more computationally concentrated). Eikelboom et al. discovered that wavelet pressure to $15 \mathrm{~KB}$ for computerized picture transmission was ideal when time and expenses are to be limited. For computational chance to be limited, JPEG pressure to $29 \mathrm{~KB}$ was a decent other option. 
All investigations to date utilizing telemedicine in ophthalmology have not stretched out the computerized interaction to advanced picture scientific procedures. This might be not easy because of the requirement for generally high-goal pictures to perform quantitative automated picture investigation. Transmission of such high-goal images is at present unfeasible for telemedicine. Notwithstanding, picture pressure calculations are, as of now, actually developing. With improved innovation, it very well might be feasible to send high-goal pictures adequately to empower advanced picture investigation.

The retinal images various process explained

\section{A. Preprocessing on Retina Image}

The main issue experienced by clinical imaging frameworks is the twisting of visual signs acquired because of defective procurement and transmission mistakes. Visible bending may emerge because of different elements like a season of openness, lighting, and development of the eye and affectability of the imaging gadgets. These influence pictures as far as differentiation, twisting and ancient rarities presented, obscure and contrast affectability. These visual changes have an adverse consequence and make the picture complex for understanding. This requires picture upgrade strategies that improve quality boundaries. Models incorporate histogram balance, picture honing, contrast change, edge upgrade and denoising. Picture preprocessing procedures include picture contrast improvement, picture commotion evacuation, edge, edge identification and picture division.

Picture pre-preparing is the essential technique of computerized fundus picture. Clamour in the clinical images has two impediments. They debase the picture quality and dark the critical data needed for exact analysis. Both have a natural effect during the investigation and must be dealt with productively. In this manner, all clinical pictures need a calculation to upgrade the image and assist the clinical professional with diagnosing and productively.

Marco et al. (2005) [6] has utilized the pay based procedure for killing the glow and difference varieties in the retinal pictures. Standardization of these varieties is performed by assessing the nonconsistency behind the scenes part of the picture. In any case, not many zones with "non-foundation" dim regions bigger than half are not influenced by this standardization. 


\section{VOLUME: 1 1, JANUARY-JUNE 2021}

Aliaa et al. (2006) [7] has introduced a similar report between different differentiation upgrade strategies for retinal pictures. These strategies are carried out on openly accessible data sets, and the outcomes are classified. These outcomes dissect the benefits and negative marks of the different differentiation upgrade strategies.

Peng et al. (2007) [8] has utilized change based strategies for edge improvement in low differentiation pictures. The yield pictures depend on the non-straight capacity, which joins the impacts of clamour. A similar examination is additionally performed with different procedures. The meaning of the red channel for precise shading retinal picture handling is clarified by Nancy et al. (2007).

Division of retinal veins is performed with red and green channels of the retinal picture. The outcomes are contrasted and the dividend yield of the green track of the retinal image. Experimental results have shown promising effects for the utilization of the red channel for retinal picture division. A close examination of the prepreparing methods in shading retinal pictures is performed by Salvatelli et al. (2007) [9].
George et al. (2008) [10] has carried out a subordinate based strategy for foundation closer view separation. The convolution of 2D Gaussian portions with the second subordinates of the info picture features the veins. However, the disadvantage of this framework is the low precision results determined in the report.

Gopal et al. (2008) [11] has proposed an area information based vein improvement strategy in shading retinal pictures. A remedy factor is gotten from the assessed corruption and used to limit the difference and iridescence variety in retinal images. A model-based vessel improvement method is proposed by Yuan et al. (2008) [12]. The foundation concealment measure, smoother 'vesselness' measure and the reactions at intersections are better than the traditional strategies. The directional field-based retinal vessel upgrade procedure is accounted for by Jian et al. (2008) [13]. A solitary advance brilliance standardization and neighbourhood improvement are utilized in this work.

Multi-scale line activity-based vein improvement is performed by Farnell et al. (2008) [14]. This multi-scale line activity calculation depends on the district developing strategy, and the outcomes are contrasted and the traditional middle 


\section{VOLUME: 1 1, JANUARY-JUNE 2021}

sifting method. The proposed approach is appropriate for all retinal infection grouping applications, and the effects likewise uncovered that the proposed way is a lot quicker than the traditional procedures.

During the past many years, numerous strategies have been proposed to deal with the clinical picture division issues utilizing solo bunching techniques. Nonetheless, the systems have a few restrictions to defeat the picture's problems, like force inhomogeneity, fractional volume impact, substantial commotions and different relics during the division cycle. Among any remaining numerical based division strategies, Fuzzy C-Means has gotten a lot of consideration since it acquires the first data from the actual picture. It doesn't need any earlier data to handle the division framework. Fluffy C-Means is the strategy for the soft bunching method. Fluffy grouping is quite possibly the main procedures in bunch investigation. Throughout the long term, there have been numerous strategies and techniques created to perform group examination. Most conventional bunch examination strategies are fresh parcelling, in which each given article is rigorously characterized into a specific gathering. Notwithstanding, practically speaking, the class ascribes of most papers are not severe and uncertain; subsequently, it isn't reasonable for hard apportioning. Favourably, the fluffy set hypothesis was proposed by Lotfi. A. Zadeh [15]. It augments the traditional set hypothesis, and it gives a valuable asset to delicate parcelling. Utilizing fluffy set hypothesis [5] for bunching is first presented by Ruspini [15]. Since fluffy bunching acquires the level of vulnerability of tests assets to each class and communicates the moderate property of their enrollments, it can all the more equitably mirror this present reality issues. As of late, the Fuzzy bunching strategy [16] is generally applied in numerous applications like the clinical conclusion, design acknowledgement, information examination and picture division. Since it doesn't need any earlier data about the objects of information and any human obstruction of images, it is a significant apparatus in dissecting pictures' conduct and underlying intricacy in clinical picture division [17]. The fluffy grouping measure is done viably by utilizing the FCM calculation [18].

B. Retinal Images template matching and its registration

A few works on layout coordinating of retinal pictures depend on more broad picture enrollment techniques, extensively 


\section{VOLUME: 1 1, JANUARY-JUNE 2021}

concentrated as of late. Notwithstanding, general retina enrollment techniques centre around coordinating with picture sets with an enormous FOV with neighbourhood disfigurements or diverse picture modalities. The current retinal format corresponding with calculations are restricted to identifying explicit articles from the picture. The layout consistently contains a specific element, like the optic circle, exudate and antiques [19]-[20].

Retinal picture enrollment itself is testing: the nonvascular surface of the retina is homogeneous in solid retinas while displaying an assortment of pathologies in undesirable retinas [21]. Retina pictures caught by connector based optics give fewer data and have low picture quality, further expanding the trouble of layout coordinating. It is informative to present current retina picture enlistment techniques, which can use for format corresponding and their achievability intending to our expressed issue. Retina picture enlistment approaches can be grouped into territory based and include based techniques. Highlight based strategies upgrade the correspondence between removed notable items in retina pictures [21]. Regularly, bifurcations, fovea, and the optic circle are essential highlights utilized for retinal picture enrollment. A little FOV layout has little likelihood of containing explicit tourist spots on the retina. Subsequently, the fovea and optic circle are not appropriate. Vascular bifurcations are more regular, while correspondingly, the modest number of bifurcations in the format can't shape the premise of a hearty enrollment. Also, the extraction of the vascular organization in low-quality pictures is troublesome. It can cause questionable vascular bearings while marking the bifurcations. General element point-based methodologies are additionally executed in retina enlistment, for example, SIFT-based [22] and SURF based techniques [23]. These methodologies can enrol the pictures in complex situations and are computationally proficient. They accept the element point sets can be dependably distinguished and coordinated to appraise the change. Albeit achievable by and large, the interaction can fizzle on bad quality retina pictures without definite highlights. Territory based methodologies match the force contrasts of a picture pair under a similitude measure, like SSD (amount of squared differences) [24], CC (CrossCorrelation) [25] and MI (common data) [26], at that point improve the comparability measure via looking in the change space. Staying away from pixellevel element recognition, such methodologies are more hearty to low- 
quality pictures than include based methods. Be that as it may, retina pictures with meagre highlights and comparable foundations will probably lead the advancement into neighbourhood extrema.

Since ophthalmology is mainly subject to visual data, it is an ideal claim to fame for telemedicine [27]. Advanced catch of pictures and the potential for transmission of these pictures through electronic exchange across enormous distances with resulting picture examination offers adequate utilization of clinical assets in massive, rustic networks that may somehow experience issues getting wellqualified assessment [28]. The most widely recognized framework used in 'teleophthalmology is "store-and-forward", where pictures are caught and later sent electronically to be examined later. These differentiations with live videoconferencing, which is right now restricted by electronic transmission rates. "Teleophthalmology" could be used between essential medical services experts, optometrists and ophthalmic trained professionals, or between various ophthalmic units. Telemedicine has even been utilized to help clinical jail officials diagnose ophthalmic objections, diminishing expenses and possible inconveniences of detainees going to expert clinical focuses [29]. In a communitarian worldwide venture,

telemedicine is financially savvy in reducing the weight of eye sickness. More extravagant nations may help limit working in the medical services frameworks of less fortunate countries. Nations with enormous spaces of meagerly populated networks, for example, Canada, Australia and India, may significantly profit by medical care conveyance to these spaces.

Telemedicine has a possible part in diabetic screening. Kawasaki et al. (2003) [30] report that 1076 of 1170 eyes' fundal pictures were effectively assessed by a specialist ophthalmologist when moved images through electronic mail. Lin et al. (2002) [31] report single non-mydriatic monochromatic wide-field computerized photography of the circle and macula to be more delicate for diabetic retinopathy screening mydriatic ophthalmoscopy when communicated electronically to an understanding site. When mediated by standard seven-field shading photos, the higher affectability of advanced photography mirrored the diminished affectability of ophthalmoscopy in distinguishing early retinopathy. TOSCA (Tele-Ophthalmological Services CitizenCentered Application) was created in Europe as a task to decrease the rate of visual impairment brought about by 


\section{VOLUME: 1 1, JANUARY-JUNE 2021}

diabetic retinopathy (Luzio et al., 2004)

[32]. Telemedicine has been investigated in evaluating for ROP. Yen et al. (2002) [33] discovered RetCam (Massie Research Laboratories, Inc., Dublin, CA) pictures caught by a neonatal attendant contrasted well and assessments performed by an accomplished ophthalmologist with great affectability, however, just reasonable particularity.

Furthermore, teleophthalmology has been used in macular sicknesses.

Eikelboom et al. (2000) [34] report on JPEG and wavelet advanced picture pressure on the nature of pictures for telemedicine. JPEG picture pressure breaks the image into squares of $8 \times 8$ pixels and converts these squares into spatial recurrence parts. Inspecting this recurrence space data by intently safeguarding the low-recurrence details and approximating the high-recurrence segments is performed. The measure of data disposed of decides the measurement of pressure. Wavelet utilizes band channels and low pass channels to the pixel lines and features of a picture. This produces data on the low-recurrence segments of the image and the even, vertical and inclining subtlety in the picture (and is all the more computationally concentrated). Eikelboom et al. discovered that wavelet pressure to $15 \mathrm{~KB}$ for computerized picture transmission was ideal when time and expenses are to be limited. For computational chance to be limited, JPEG pressure to $29 \mathrm{~KB}$ was a decent other option.

All investigations to date utilizing telemedicine in ophthalmology have not stretched out the computerized interaction to advanced picture scientific procedures. This might be not easy because of the requirement for generally high-goal pictures to perform quantitative automated picture investigation. Transmission of such high-goal images is at present unfeasible for telemedicine. Notwithstanding, picture pressure calculations are, as of now, actually developing. With improved innovation, it very well might be feasible to send high-goal pictures adequately to empower advanced picture investigation.

\section{CONCLUSIONS}

The division is the above all else prerequisite for the retinal veins of late and quickly developing exploration territory. The exact and successful concentrate of retinal vasculature vb's gives many modernized frameworks in recognition, extraction and ID of oculist sickness. As hundred's of promising strategies at present accessible, need for development and advance the fragment ways. The survey calculations add to aetiology, and 


\section{VOLUME: 1 1, JANUARY-JUNE 2021}

obsessive and commotion contained retinal

eye pictures are fundamental for examining retinal fundus shading pictures.

Previously mentioned courses in this audit are analyzed with datasets or data sets with, at any rate, ten images each from the DRIVEs and STAREs DB's. Few pictures of specific boundaries determine the actions for execution in the majority of the papers. The photos in the DRIVE and STARE data sets. Applying the aboveevaluated techniques to various fundus pictures taken by multiple goal fundus cameras under numerous other homestead conditions is a current region for reality finding in vein annihilation calculations.

\section{FUTURE SCOPE}

The methods assessed can be reached out to cutting edge procedures applied to new data sets, including HD and nearby data sets. Can survey them dependent on various boundaries considered for assessment of results.

Table 1: Comparison methods of Different Approaches

\begin{tabular}{|l|l|l|l|l|}
\hline Method & Source & Sensitivity(\%) & Specificity(\%) & Accuracy(\%) \\
\hline observer & DRIVEdatabase & 77.63 & 97.23 & 94.7 \\
\hline observer & STAREdatabase & 89.50 & 93.84 & 93.4 \\
\hline Sinthanayothin[10] & Local database & 83.30 & 91.00 & ------ \\
\hline Abramoff[11] & DRIVEdatabase & 71.45 & 94.16 & 92.9 \\
\hline Staal[12] & DRIVEdatabase & 94.42 & 95.2 & --- \\
\hline Staal[12]- & STARE database & 95.16 & 96.14 & ---- \\
\hline Soares[13] & DRIVEdatabase & 94.66 & 96.14 & ---- \\
\hline Soares[13] & STARE database & 94.80 & 96.71 & ---- \\
\hline Ricci, Perfetti[14] & DRIVEdatabase & 95.63 & 95.58 & ---- \\
\hline Ricci, Perfetti[14] & STARE database & 95.84 & 96.02 & ---- \\
\hline
\end{tabular}

\section{REFERENCES}

[1]. B. JL. "Photo physical Processes in Recent Medical Laser Developments". Lasers Med Sci., vol. 1, pp.47-66. 1986.

[2]. B. GM. "Lasers in Medicine and Surgery”, JAMA, vol. 256, pp. 900-907, 1986.

[3]. N. M. Bressler, S.B. Bressler, and E.S. Gragoudas, "Clincal characteristics of choroidal neovascular membranes,” Arch. Ophthalmol., vol. 105, pp. 209-213, 1987. 


\section{VOLUME: 11 , JANUARY-JUNE 2021}

[4]. P. N. Monahan, K. A. Gitter, and G. Cohen, "Evaluation of Persistence of Subretinal Neovascular Membranes Using Digitized Angiographic Analysis," Retina-J. Retinal, Vitreous Diseases, vol. 13, pp. 196-201, 1993.

[5]. S. Fine, "Observations Following Laser Treatment for Choroidal Neovascularization," Arch. Ophthalm., vol. 106, pp. 1524-1525, 1988.

[6]. Marco Foracchia, Enrico Grison and Alfredo Ruggeri, 'Luminosity and Contrast Normalization in Retinal Images', Medical Image Analysis (Elsevier), Vol. 9, 2005, pp 179190.

[7]. Youssif, Aliaa \& Ghalwash, Atef \& Ghoneim, Amr. (2006). Comparative Study of Contrast Enhancement and Illumination Equalization Methods for Retinal Vasculature Segmentation. Cairo International Biomedical Engineering Conference (CIBEC).

[8]. Peng Feng, Ying-jun Pan, Biao Wei, Wei Jin and Deling Mi , 'Enhancing Retinal Image by the Contourlet Transform', Pattern Recognition Letters ( Elsevier), Vol. 28, 2007, pp 516522.

[9]. Salvatelli A., Bizai G., Barbosa G., Drozdowicz and Delrieux , 'A Comparative Analysis of Pre-processing Techniques in Color Retinal Images', Journal of Physics: Conference series 90,2007

[10]. George K.M., Pantelis A.A., Konstantinos K.D., Nikolaos A.M., Thierry G.Z., 'Detection of Glaucomatous Change Based on Vessel Shape Analysis', Computerized Medical Imaging and Graphics, Vol. 32, 2008, pp 183-192.

[11]. Joshi, Gopal Datt, and Jayanthi Sivaswamy. "Colour retinal image enhancement based on domain knowledge." 2008 Sixth Indian Conference on Computer Vision, Graphics \& Image Processing. IEEE, 2008.

[12]. Yuan, Y., \& Chung, A. C. (2008, August). Multi-scale model-based vessel enhancement using local line integrals. In 2008 30th Annual International Conference of the IEEE Engineering in Medicine and Biology Society (pp. 2225-2228). IEEE.

[13]. Jian Chen, Jie Tian, Zichun Tang, Jian Xue, Yakang Dai, and Jian Zheng. "Retinal vessel enhancement and extraction based on directional field." Journal of X-Ray Science and Technology 16, no. 3 (2008): 189-201. 


\section{VOLUME: 11 , JANUARY-JUNE 2021}

[14]. Farnell, Damian JJ, F. N. Hatfield, P. Knox, M. Reakes, S. Spencer, D. Parry, and Simon P. Harding. "Enhancement of blood vessels in digital fundus photographs via the application of multiscale line operators." Journal of the Franklin institute345, no. 7 (2008): 748-765.

[15]. Zadeh L.A, Fuzzy sets. Inf. Control (8), (1965), pp. 338-353.

[16]. A Sathya, Anudevi Samuel, and M.S. Sheeba, Robust Fuzzy C-Means based Minimal Spanning tree method For Segmentation of Breast MRI, International Conference on Mathematical Sciences, Elsevier Publications, (2014) pp. 495-501.

[17]. Ye Xing et al., Simultaneous Estimation and Segmentation of T1 Map for Breast parenchyma Measurement, 4th IEEE International Symposium on Biomedical Imaging, (2007),pp. $332-335$.

[18]. S. Ramathilagam et al., Journal of Intelligent and Fuzzy Systems, (2014) 27(5): 25732595.

[19]. X. Zhang, G. Thibault, E. Decenci'ere, B. Marcotegui, B. La“y, R. Danno, G. Cazuguel, G. Quellec, M. Lamard, P. Massin et al., "Exudate detection in color retinal images for mass screening of diabetic retinopathy,"Medical image analysis, vol. 18, no. 7, pp. 1026-1043, 2014.

[20]. A. D. Mora, J. Soares, and J. M. Fonseca, "A template matching technique for artifacts detection in retinal images," in Image and Signal Processing and Analysis (ISPA), 2013 8th International Symposium on. IEEE, 2013, pp. 717-722.

[21]. C. V. Stewart, C.-L. Tsai, and B. Roysam, "The dual-bootstrap iterative closest point algorithm with application to retinal image registration," IEEE transactions on medical imaging, vol. 22, no. 11, pp. 1379-1394, 2003.

[22]. Y. Wang, J. Shen, W. Liao, and L. Zhou, “Automatic fundus images mosaic based on sift feature," in Image and Signal Processing (CISP), 2010 3rd International Congress on, vol. 6. IEEE, 2010, pp. 2747-2751.

[23]. C. Hernandez-Matas, X. Zabulis, and A. A. Argyros, "Retinal image registration based on keypoint correspondences, spherical eye modeling and camera pose estimation," in Engineering in Medicine and Biology Society (EMBC), 2015 37th Annual International Conference of the IEEE. IEEE, 2015, pp. 5650-5654. 


\section{VOLUME: 11 , JANUARY-JUNE 2021}

[24]. K. J. Friston, J. Ashburner, C. D. Frith, J.-B. Poline, J. D. Heather, and R. S. Frackowiak, "Spatial registration and normalization of images," Human brain mapping, vol. 3, no. 3, pp. 165-189, 1995.

[25]. A. V. Cideciyan, "Registration of ocular fundus images: an algorithm using crosscorrelation of triple invariant image descriptors," IEEE Engineering in Medicine and Biology Magazine, vol. 14, no. 1, pp. 52-58, 1995.

[26]. Y.-M. Zhu, "Mutual information-based registration of temporal and stereo retinal images using constrained optimization," Computer methods and programs in biomedicine, vol. 86, no. 3, pp. 210-215, 2007.

[27]. Lamminen et al., 2003 H. Lamminen, V. Voipio, K. Ruohonen and H. Uusitalo, Telemedicine in ophthalmology, Acta Ophthalmol. Scand. 81 (2003), pp. 105-109. AbstractMEDLINE | Abstract-EMBASE | Full Text via CrossRef

[28]. Yogesan et al., 2000 K. Yogesan, M. Cuypers, C. Barry, I. Constable and L. Jitskaia, Tele-ophthalmology screening for retinal and anterior segment diseases, J. Telemed. Telecare 6 (2000) (Suppl. 1), pp. S96-S98. Abstract-MEDLINE

[29]. Yogesan et al., 2001 K. Yogesan, C. Henderson, C. Barry and I. Constable, Online eye care in prisons in Western Australia, J. Telemed. Telecare 7 (2001) (Suppl. 2), pp. 63-64.

[30]. Kawasaki et al., 2003 S. Kawasaki, S. Ito, Y. Mori, T. Saito, H. Fukushima, S. Kato and H. Sekihara, Use of telemedicine in periodic screening of diabetic retinopathy, Telemed. J. E Health 9 (2003), pp. 235-239.

[31]. Lin et al., 2002 D. Lin, M. Blumenkranz, R. Brothers and D. Grosvenor, The sensitivity and specificity of single-field nonmydriatic monochromatic digital fundus photography with remote image interpretation for diabetic retinopathy screening: a comparison with ophthalmoscopy and standardized mydriatic color photography, Am. J. Ophthalmol. 134 (2002), pp. 204-213. SummaryPlus | Full Text + Links | PDF (175 K)

[32]. Luzio et al., 2004 S. Luzio, S. Hatcher, G. Zahlmann, L. Mazik, M. Morgan, B. Liesenfeld, T. Bek, H. Schuell, S. Schneider and D. Owens et al., Feasibility of using the TOSCA

telescreening procedures for diabetic retinopathy, Diabet. Med. 21 (2004), pp. 1121-1128. 


\section{VOLUME: 1 1, JANUARY-JUNE 2021}

[33]. Yen et al., 2002 K. Yen, D. Hess, B. Burke, R. Johnson, W. Feuer and J. Flynn, Telephotoscreening to detect retinopathy of prematurity: preliminary study of the optimum time to employ digital fundus camera imaging to detect ROP, J. AAPOS 6 (2002), pp. 64-70. Abstract | PDF (174 K)

[34]. Eikelboom et al., 2000 R. Eikelboom, K. Yogesan, C. Barry, I. Constable, M. TayKearney, L. Jitskaia and P. House, Methods and limits of digital image compression of retinal images for telemedicine, Invest. Ophthalmol. Vis. Sci. 41 (2000), pp. 1916-1924. 